\title{
Alteration of FOXM1 expression and macrophage polarization in refractory meningiomas during long-term follow-up
}

\author{
Jun Takei $^{1,2}$, Toshihide Tanaka ${ }^{1}$, Akihiko Teshigawara ${ }^{1}$, Satoru Tochigi ${ }^{1}$, Yuzuru Hasegawa ${ }^{1}$, \\ Yuichi Murayama \\ ${ }^{1}$ Department of Neurosurgery, Jikei University School of Medicine Kashiwa Hospital, Chiba, Japan; ${ }^{2}$ Department of Neurosurgery, Jikei University \\ School of Medicine, Tokyo, Japan \\ Correspondence to: Toshihide Tanaka, MD, PhD. Department of Neurosurgery, Jikei University School of Medicine Kashiwa Hospital, 163-1 Kashiwa- \\ shita, Kashiwa, Chiba 277-8567, Japan. Email: ttanaka@jikei.ac.jp.
}

\begin{abstract}
Malignant progression of grade I meningioma with a long latency period is rare. We experienced grade II/III meningiomas with refractoriness and recurrence from grade I meningiomas through multiple surgeries. Three patients with atypical/anaplastic meningioma experienced long-latent recurrence after initial surgery for grade I (meningothelial) meningioma without following adjuvant radiotherapy were included in the present study. Histological findings of the initial tumors in all cases (case 1, 2, and 3) revealed meningothelial meningioma with $1 \%$, 5\%, and $0.1 \%$ MIB-1 positive cells, respectively. Surprisingly, magnetic resonance imaging (MRI) detected a recurrent tumor 2, 12, and 12 years after the initial operation, respectively. Case 1 was atypical meningioma after third recurrence, and case 2 and 3 were anaplastic meningioma after second and third recurrence, respectively. The patient in case 2 received adjuvant radiotherapy. In case 2, the tumor recurred intracranial and distant metastasis to the lung with huge substantial pleural effusion was detected. To investigate the pathogenesis of malignant progression from benign to malignant meningioma, CD163/CD68 expression by immunohistochemically and FOXM1 mRNA expression by RT-PCR were compared using surgical specimens from initial and recurrent tumors in all three patients. The ratio of CD163/CD68 positivity and FOXM1 mRNA expression were increased in recurrent tumors compared with matched initial tumors. CD163 and FOXM1 expression levels were induced even in recurrent grade I meningioma, suggesting that macrophage polarization and pro-mitotic transcriptional factor might be associated with clinical behavior of meningioma and be useful as a prediction marker for malignant progression. Careful long-term follow-up is important for early diagnosis of malignant progression in meningiomas, even if grade I meningioma is completely resected. Development of a multidisciplinary approach including radiation and novel molecular targeted therapy is expected for recurrent and malignant meningiomas.
\end{abstract}

Keywords: Anaplastic meningioma; malignant progression; FOXM1; macrophage polarization; long-term follow-up

Submitted Apr 21, 2020. Accepted for publication Oct 30, 2020.

doi: $10.21037 /$ tcr-20-1896

View this article at: http://dx.doi.org/10.21037/tcr-20-1896

$\wedge$ ORCID: 0000-0002-6474-6317. 


\section{Introduction}

Surgical resection and radiation are the major therapeutic modalities for meningioma. However, meningiomas frequently recur regardless of macroscopically complete removal of the tumor at a rate of approximately $19 \%$ within 10 years (1). Meningioma with increased mitotic activity, hypercellularity, macronuclei, and necrosis is defined as atypical or anaplastic meningioma. Anaplastic meningioma is a rare type of malignant meningioma, accounting for less than $5 \%$ of all meningiomas (2). Once benign grade I meningioma was progressed malignantly, aggressive salvage surgery followed by intensive radiotherapy including gamma knife and cyberknife are treatment strategies. There are currently no effective adjuvant chemotherapies, including cytotoxic chemotherapy and anti-vascular endothelial growth factor (VEGF) therapy such as bevacizumab, for recurrent malignant meningiomas after surgery and radiation therapy $(3,4)$. Patients cannot help undergo multiple craniotomies for recurrent meningiomas, and unfortunately the patient's quality of life tends to decline following multiple operations.

Even benign grade I meningioma sometimes shows recurrence and malignant progression, however, a latency period of more than 10 years followed by malignant progression from grade I to grade III is very rare, and extremely difficult to predict in advance. To understand the pathogenesis of refractory meningiomas, the genomic mutations associated with progression of meningioma are demonstrated. Inactivation of NF2 is the most frequently detected. Recent studies also have found mutations in several other genes as oncogenic drivers such as AKT, SMO, TRAF7, CDKN2, TERT, and POLR2A in atypical and anaplastic meningiomas (5-8). In meningiomas in malignant progression with de novo and secondary progression, telomerase reverse transcriptase (TERT) promoter mutations were identified in recurrent meningioma in malignant progression arise from both benign and malignant tumors $(9,10)$.

Little is known about biomarkers to predict recurrence for long-term latency. In addition, it is hard to analyze the paired samples, which means primary and recurrent tumor samples, because of the rarity.

Forkhead box M1 (FOXM1), a pro-mitotic transcription factor, was identified from meningioma samples by RNA sequencing, whole-exome sequencing, and immunohistochemistry (11). FOXM1 is highly expressed in tumors, particularly invasive tumors (12). FOXM1 regulates angiogenesis via expression of vascular endothelial growth factor, invasion via matrix metalloproteinase, survival, cell cycle, tumor cell migration, and DNA damage responses in many tumor cell types (13), Although FOXM1 has been associated with worse clinical outcomes as a marker for aggressive meningioma, the clinical significance of FOXM1 in grade I meningioma progression, and alteration of FOXM1 during malignant progression in same patients has not been clearly demonstrated.

Moreover, a greater comprehension of immunological regulation of the tumor microenvironment might aid in the development of therapeutic modalities for meningiomas. Immunological constituents including tumor-infiltrating lymphocytes, natural killer cells, dendritic cells, and macrophages are critical to the tumor microenvironment. Recent studies demonstrated that presence of these cells in tumor specimens determined by immunohistochemistry affected their potential impact on clinical prognosis (14). Macrophages have a dual role in tumor immunity and express different functional programs in response to microenvironment signals, defined as M1-M2 polarization (15). To assess the M2-polarized tumorassociated macrophages (TAM) as prognostic value, CD163 is used as a marker of TAM and an indicator of a worse prognosis in various cancers (16-21). However, the role of M1-M2 polarization in prognosis of refractory and highgrade meningiomas has not been elucidated.

Here, we report three cases of recurrent meningioma with malignant progression during treatment comprising multiple craniotomies over a long-term duration. To explore reliable predictive biomarker for malignant progression of benign meningioma via long-term follow-up, M1-M2 macrophage polarization and FOXM1 expression were compared by using paired samples of initial and recurrent tumors from the same patients. We present the following article in accordance with the AME Case Series reporting checklist (available at http:// dx.doi.org/10.21037/tcr-20-1896).

\section{Materials \& methods}

\section{Ethics}

The present study was approved by the institutional review board of our institute [permission number; 15-105 and 30137(9158)]. All procedures performed in studies involving human participants were in accordance with the ethical standards of the institutional and/or national research committee(s) and with the Helsinki Declaration (as revised 
in 2013). Written informed consent was obtained from the all patients.

\section{Study design and setting}

This study was a single-center case series and retrospective analysis of 3 non-consecutive patients with benign meningioma who were performed multiple craniotomies at an academic hospital in Japan between 2005 and 2020.

\section{Immunobistochemical analyses}

Immunohistochemical analyses were performed on 4- $\mu \mathrm{m}$ sections of formalin-fixed, paraffin-embedded (FFPE) tissue from 12 tumors. Sections were stained with antiCD68 antibody (1:400, \#76437, CST), anti-CD163 antibody (1:500, \#93498, CST), and anti-FOXM1 antibody (1:250, ab207298, abcam). Antigen retrieval was implemented in pH 6.0 citrated buffer using an autoclave. Immunohistochemical staining was assessed by two authors (JT, TT) with blinded clinical information and the results of the consensus were reported.

Immunohistochemical findings were assessed as previously described (22). For CD68, CD163 and FOXM1 evaluation, stained sections were screened at a low-power field $(40 \times)$ and 5 hot spots were selected. Then, the number of positive cells in these areas was enumerated at a highpower field (HPF: 400×, $0.47 \mathrm{~mm}^{2}$ ) using Fiji (23).

\section{Quantitative real-time reverse transcription polymerase chain reaction (RT-PCR) analysis}

For messenger RNA (mRNA) expression analysis, total RNA was isolated using Allprep DNA/RNA FFPE Kit (QIAGEN, Hilden, Germany) from FFPE sections and reverse transcribed using PrimeScript RT Master Mix (Takara Bio Inc., Shiga, Japan), according to the manufacturer's protocol. Real-time amplification was achieved using the QuantStudio 5 Real-Time PCR System (Applied Biosystems, Weisterstadt, Germany). mRNA expression was then analyzed by TaqMan Gene Expression Assays (ACTB, Hs01060665_g1 as an internal control; FOXM1, Hs01073586_m1 as a primer of a target gene) (Applied Biosystems, Weiterstadt, Germany). PCR was perfumed as follows: denaturation for $10 \mathrm{~min}$ at $95{ }^{\circ} \mathrm{C}$, followed by 40 cycles at $95^{\circ} \mathrm{C}$ for $15 \mathrm{sec}$ and $60^{\circ} \mathrm{C}$ for $1 \mathrm{~min}$. FOXM1 mRNA expression was measured by the $\Delta \Delta \mathrm{CT}$ method.

\section{Case presentation}

\section{Case 1 (KS01)}

A 65-year-old woman presented with headache and motor apraxia. MRI showed an extra-axial tumor in the left parietal convexity showing hyperintense signals on T1and $\mathrm{T} 2$-weighted images with homogenous gadolinium enhancement with slight edema (Figure $1 A, B)$. The tumor tissue was elastic and hard with vasculature and en plaque dural attachment. The tumor was totally removed, and histological diagnosis was meningothelial meningioma (MIB-1 index: 1\%) (Figure 1C). The postoperative course was uneventful.

However, the patient suffered from abnormal behavior and epilepsy two years after the initial operation. MRI demonstrated a local recurrent tumor appearing as hyperintense signals on T1- and T2-weighted images with heterogeneous gadolinium enhancement (data not shown). The patient underwent a second craniotomy. The mass was attached tightly to the falx and the lateral wall of the superior sagittal sinus (SSS). A hypervascular tumor, which was identified just beneath the dura with fragile tumor vessels, was removed by en bloc resection, and the residual tumor attached to the SSS was removed by piecemeal resection. The dura was closed using Gore-Tex mesh (W.L. Gore \& Associates, Inc., Newark, DE, USA). Histological diagnosis was meningothelial meningioma (MIB-1 index: 5\%).

One year after the operation, the patient suffered from expressive aphasia and paresthesia of the right lower extremity. MRI demonstrated local recurrence of the tumor, which invaded the skull and anterior and posterior dura. The third operation was subsequently carried out, and the gross appearance of this tumor tissue differed from tumor tissue obtained from the previous surgery. The tumor was totally removed except for cancerous tissue attached to the SSS. The dura and the skull involving the tumor were also removed by cranioplasty and duraplasty. Histological findings of the tumor revealed malignant change from meningothelial to atypical meningioma without adjuvant radiotherapy (MIB-1 index: 10\%) (Table 1).

Nine months after the operation, the residual tumor recurred, and the fourth operation was carried out. The tumor invaded into the contralateral side via the falx. Histological diagnosis was atypical meningioma (MIB-1 index: 10\%) (Table 1). Six months after the fourth operation, the patient suffered from right hemiparesis and total aphasia. MRI revealed that the tumor aggressively recurred not only into the left parietal convexity with invasion to 

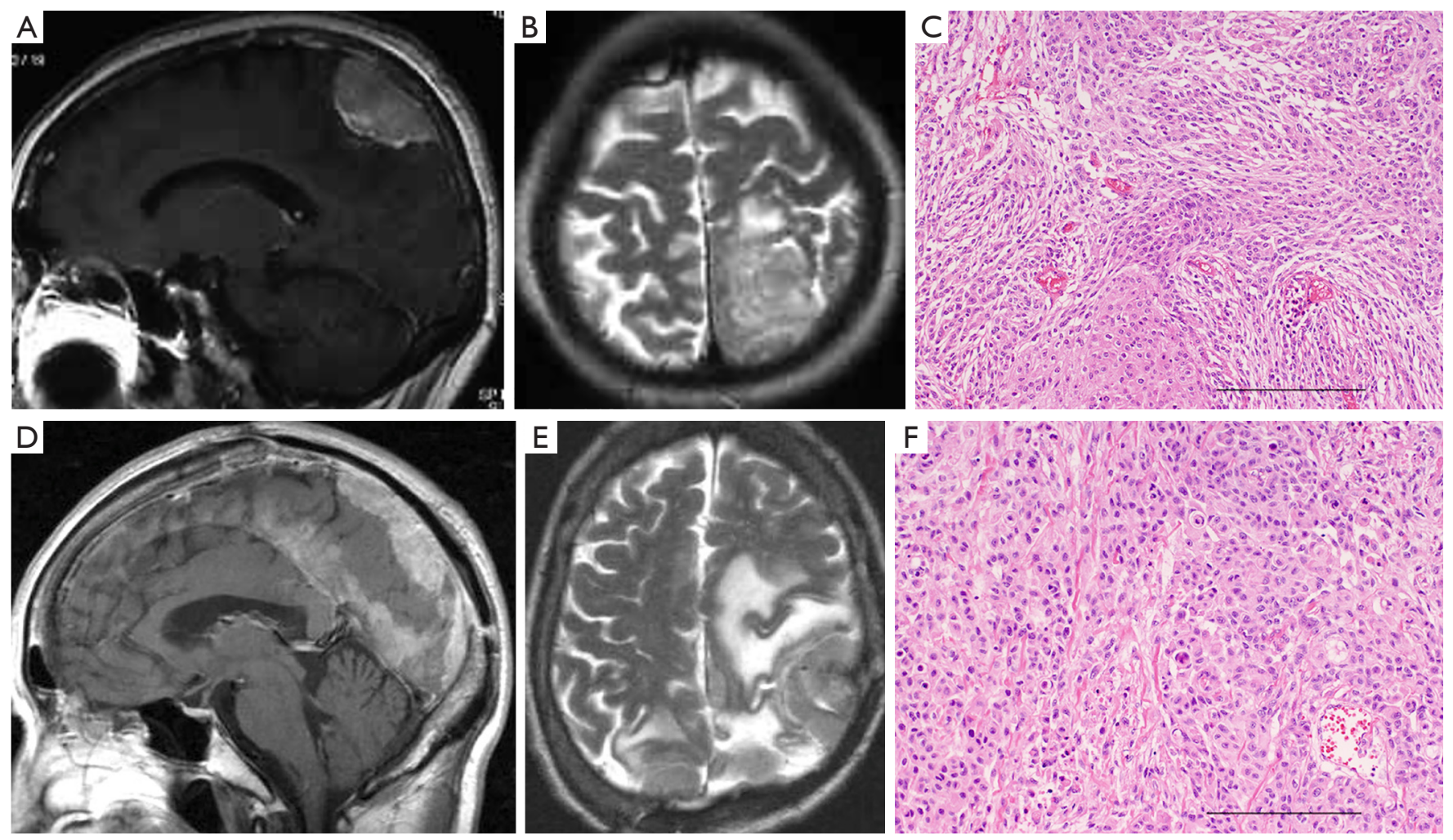

Figure 1 Preoperative magnetic resonance imaging (MRI) and histological findings of KS01 (case 1). Preoperative gadolinium-enhanced MRI (A) and T2-weighted MRI (B) at the initial stage (KS01A) showing a homogenous enhanced tumor in the right parietal lobe. Preoperative gadolinium-enhanced MRI (C) and T2-weighted MRI (D) at the third recurrence (KS01C) showing an en plaque tumor in the right parietal and occipital lobe with skull invasion. Histological findings of the initial tumor (KS01A) (E). Tumor cells were arranged in a sheet with high cellularity and mitotic cells along with focal necrosis and diagnosed as meningothelial meningioma (WHO grade I) (hematoxylin \& eosin staining: $200 \times$, scale bar $=200 \mu \mathrm{m}$ ). (F) Histological imaging at the third recurrence revealing high cellularity with mitotic cells (atypical meningioma; WHO grade II) (hematoxylin \& eosin staining: $200 \times$, scale bar $=200 \mu \mathrm{m}$ ).

the SSS but also extending to the right convexity via the falx and the tentorial edge and the transverse sinus on the left side (Figure 1D,E). To remove the mass effect, the tumor in the convexity and the falx was removed in the fifth operation. However, the tumor extending along the tentorial edge was not removed. Histological diagnosis was atypical meningioma (MIB-1 index: 10\%) (Figure $1 F$, Table 1). Two years after operation, she passed away due to liver metastases from uterus cancer. The autopsy was not obtained.

\section{Case 2 (KSO2)}

A 61-year-old woman presented with motor aphasia and mild hemiparesis on the right side. MRI showed an en plaque lesion that appeared hyper- and hypointense on T1- and $\mathrm{T} 2$-weighted images with heterogeneous gadolinium enhancement (Figure 2A,B). The tumor including the dura adjacent to the tumor was totally removed, and duraplasty with Gore-Tex was carried out. Histological diagnosis was meningothelial meningioma (MIB-1 index: 5\%) (Figure 2C). The postoperative course was uneventful. Follow-up MRI for 6 years after surgery detected no recurrence. The patient then discontinued follow-up for 6 years. However, she suffered from gait disturbance, loss of activity, and paresthesia of the right extremities 12 years after the initial operation. MRI demonstrated a de novo tumor with two components appearing hyper- and isointense on T1and $\mathrm{T} 2$-weighted images with heterogeneous gadolinium enhancement (Figure 2D,E).

The patient subsequently underwent left frontal craniotomy. The mass was located extra-axially and 


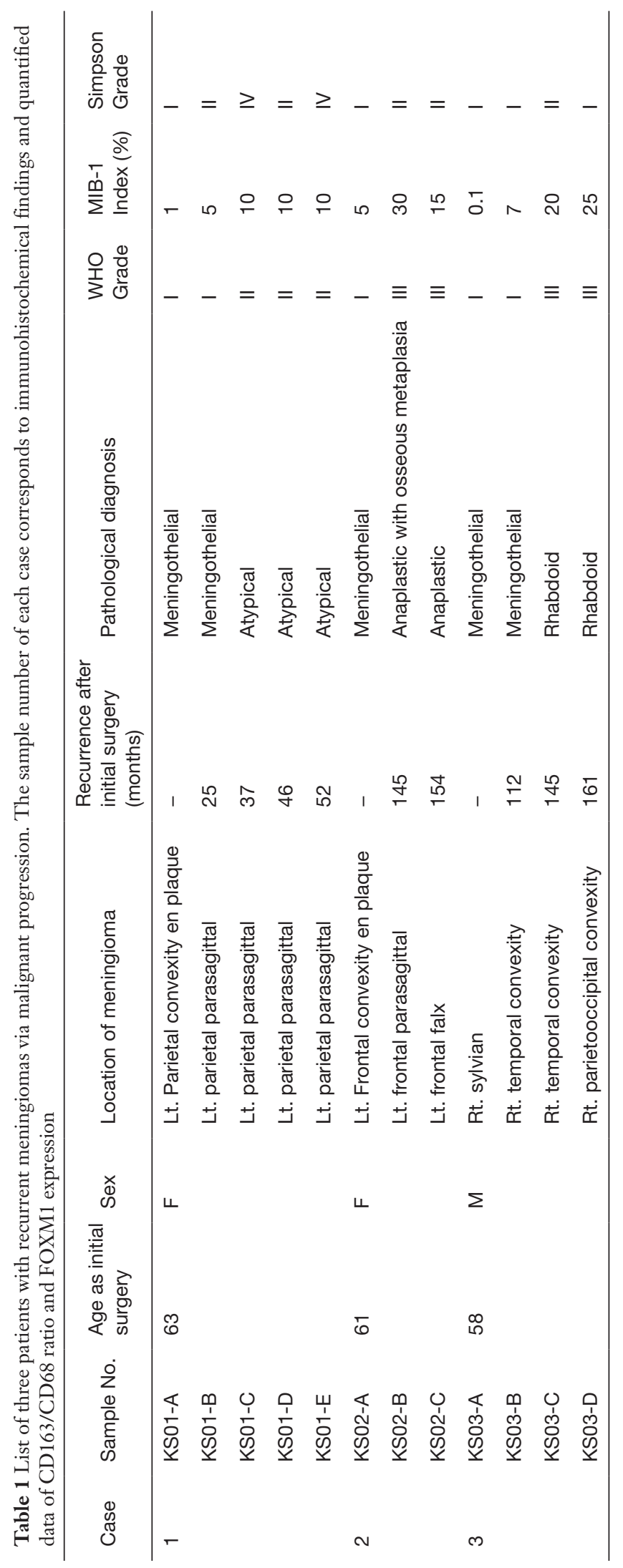

consisted of two components, which corresponded to the MRI findings. A soft and red-brownish tumor was initially identified just beneath the dura and showed easy bleeding on incision. Following removal of this surface tumor, a yellowish and elastic component of the tumor was identified just behind the red-brownish tumor. The boundaries between the tumor and the normal cortex were obscure. Biopsied specimens were taken from three regions: the red-brownish component of the tumor demonstrated homogenous nuclei of cells with sheet-like arranged and high cellularity containing circumscribed collagen fibers (Figure $2 F$ ). The yellowish tumor composed marked circumscribed collagen fibers with bone metaplasia (Figure 2G,H). Histological diagnosis was anaplastic meningioma with osseous metaplasia. After the second surgery, the patient received postoperative adjuvant radiotherapy (60 Gy/30 fr).

However, nine months after radiation, the tumor recurred at the bottom of the resection cavity in the left frontal lobe. She subsequently underwent a third surgery. The margin of the yellowish, elastic, and hard tumor attached to the thickened dura was easily identified from the normal cortex, and the tumor was totally removed by frontal lobectomy.

Six months after the third surgery, the patient suffered from dyspnea. Chest CT revealed substantial pleural effusion in the right lung because the meningioma had metastasized to the lung (Figure 2I,7). Two months after chest CT, the patient passed away. Consent for autopsy was obtained from the patient's family.

\section{Autopsy findings}

A recurrent tumor was observed in the left frontal lobe (Figure $3 A$ ), and the space created in the frontal skull base after left frontal lobectomy was completely filled with the recurrent tumor, which contained ossified tissue. The tumor disseminated into the brain stem and multiple lesions were observed on the pia of the spine and cauda equina (Figure 3B). The tumor had also metastasized to both sides of the lung, which consisted of multiple white nodules (Figure 3C) and was associated with bloody pleural effusion.

\section{Case 3 (KS03)}

A 58-year-old man presented with headache. Magnetic resonance imaging (MRI) showed a lesion along the right sylvian fissure appearing hyper- and hypointense on T1- 

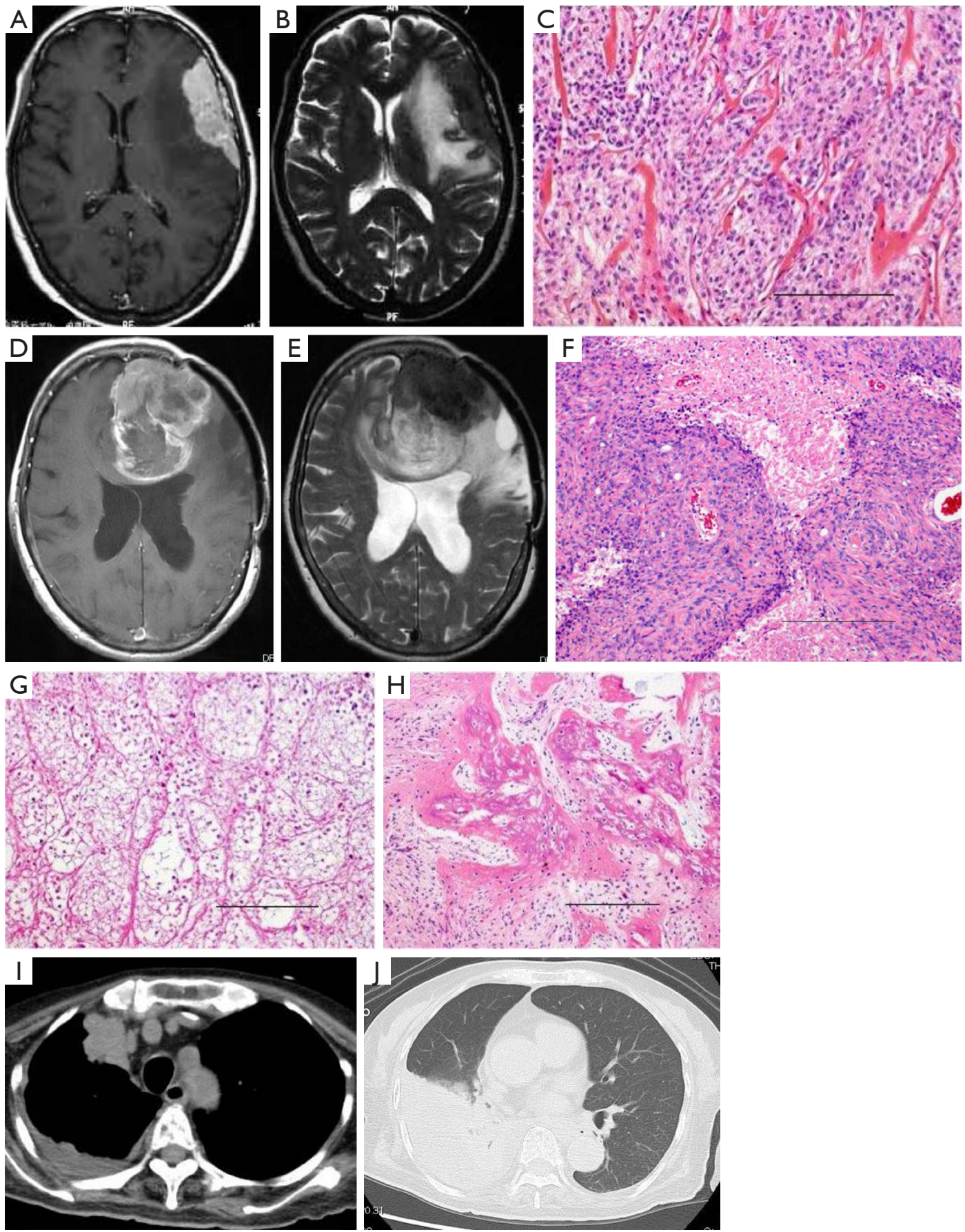

Figure 2 Preoperative magnetic resonance imaging (MRI), computed tomography (CT), and histological findings of KS02 (case 2). Preoperative gadolinium-enhanced MRI (A) and T2-weighted MRI (B) at the initial stage (KS02A) showing an enhanced en plaque tumor with perifocal edema in the left frontal lobe. Histological findings of the initial tumor (KS02A) (C). Tumor cells were arranged in a sheet with an onion-like structure and diagnosed as meningothelial meningioma (WHO grade I (hematoxylin \& eosin staining: 200x, scale bar =200 um). Gadolinium-enhanced MRI (D) and T2-weighted MRI (E) 12 years after total resection (KS02B) showing a large heterogeneously enhanced tumor associated with expanding perifocal edema in the left frontal lobe compressing the corpus callosum. Histological imaging at the third recurrence (KS02C) revealing high cellularity with mitosis and focal necrosis (anaplastic meningioma; WHO grade III) (hematoxylin \& eosin staining: 200×, scale bar =200 $\mu \mathrm{m}$ ). (F) containing osteoplastic metaplasia (hematoxylin \& eosin staining: 200x, scale bar $=200 \mu \mathrm{m})(\mathrm{G}, \mathrm{H})$. Chest CT demonstrating multiple metastatic tumors to the right lung (I), consisting of substantial pleural effusion (J). 

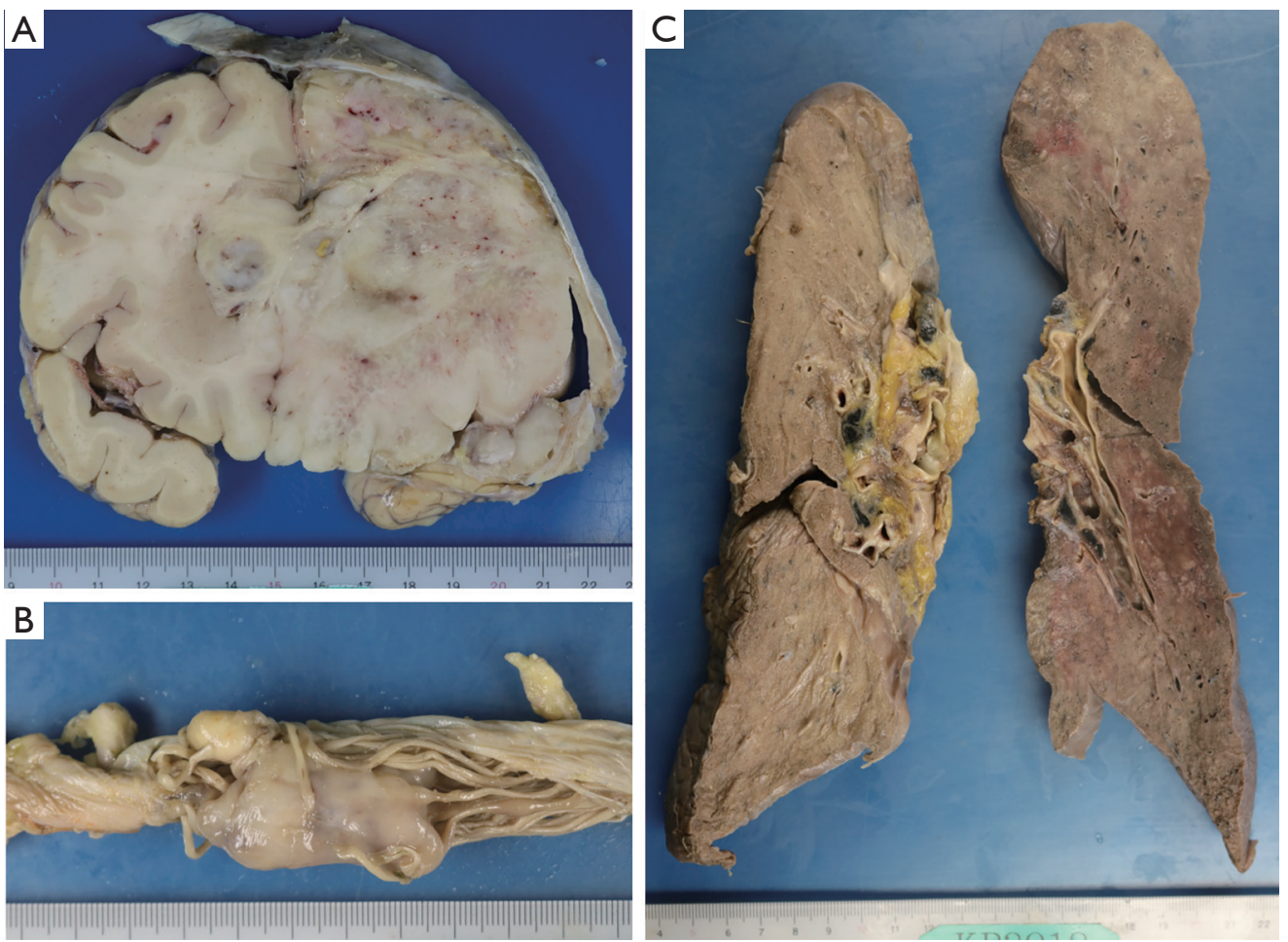

Figure 3 Macroscopic findings of autopsy. Autopsy demonstrated a recurrent tumor in the left frontal lobe invading into the basal ganglia (A) with dissemination of multiple tumors to the cauda equina (B). Metastasized tumors to the lung with multiple whitish nodules beneath the pleural space were observed (C).

and T2-weighted images with homogeneous gadolinium enhancement (data not shown). The tumor was totally removed, and histological diagnosis was meningothelial meningioma (MIB-1 index: $0.1 \%$ ) (Table 1). The postoperative course was uneventful. However, a recurrent tumor was incidentally discovered nine years after the initial operation. MRI demonstrated a recurrent tumor in the right inferior frontal gyrus with homogenous gadolinium enhancement (Figure 4A,B). The patient underwent reoperation. The tumor invading into the cortex was totally removed with duraplasty using the fascia. Histological diagnosis was meningothelial meningioma (MIB-1 index: 7\%) (Figure 4C, Table 1).

Three years after the second operation, the patient suffered from severe headache and deep coma. MRI demonstrated a huge mass in the right temporal convexity with uncal herniation (Figure 4D,E). The patient underwent emergent reoperation. After removal of the bone flap and dural incision, the tumor accordance with huge hematoma was revealed. The tumor containing the hematoma was completely removed with duraplasty using the fascia.
Histological diagnosis was rhabdoid meningioma (grade III; MIB-1 index: 20\%) (Figure 4F, Table 1). Postoperatively, the patient's consciousness was recovered without any neurological deficits.

However, he suffered from left hemianopsia and dressing apraxia 16 months after the third operation. Consequently, hemiparesis in the left extremities deteriorated. Follow-up MRI demonstrated an en plaque tumor attached to the dura and the cystic tumor with nodule in the parietal lobe on the right (Figure $4 G, H$ ). The fourth operation was carried out. The tumor was totally removed with duraplasty using the fascia. Histological diagnosis was rhabdoid meningioma (MIB-1 index: 25\%) (Table 1). Postoperatively, he received gamma knife one month after the operation.

\section{Results}

\section{Immunobistochemical analyses of CD163/CD68}

Series of surgical samples from same patients who underwent repeated multiple craniotomies during 

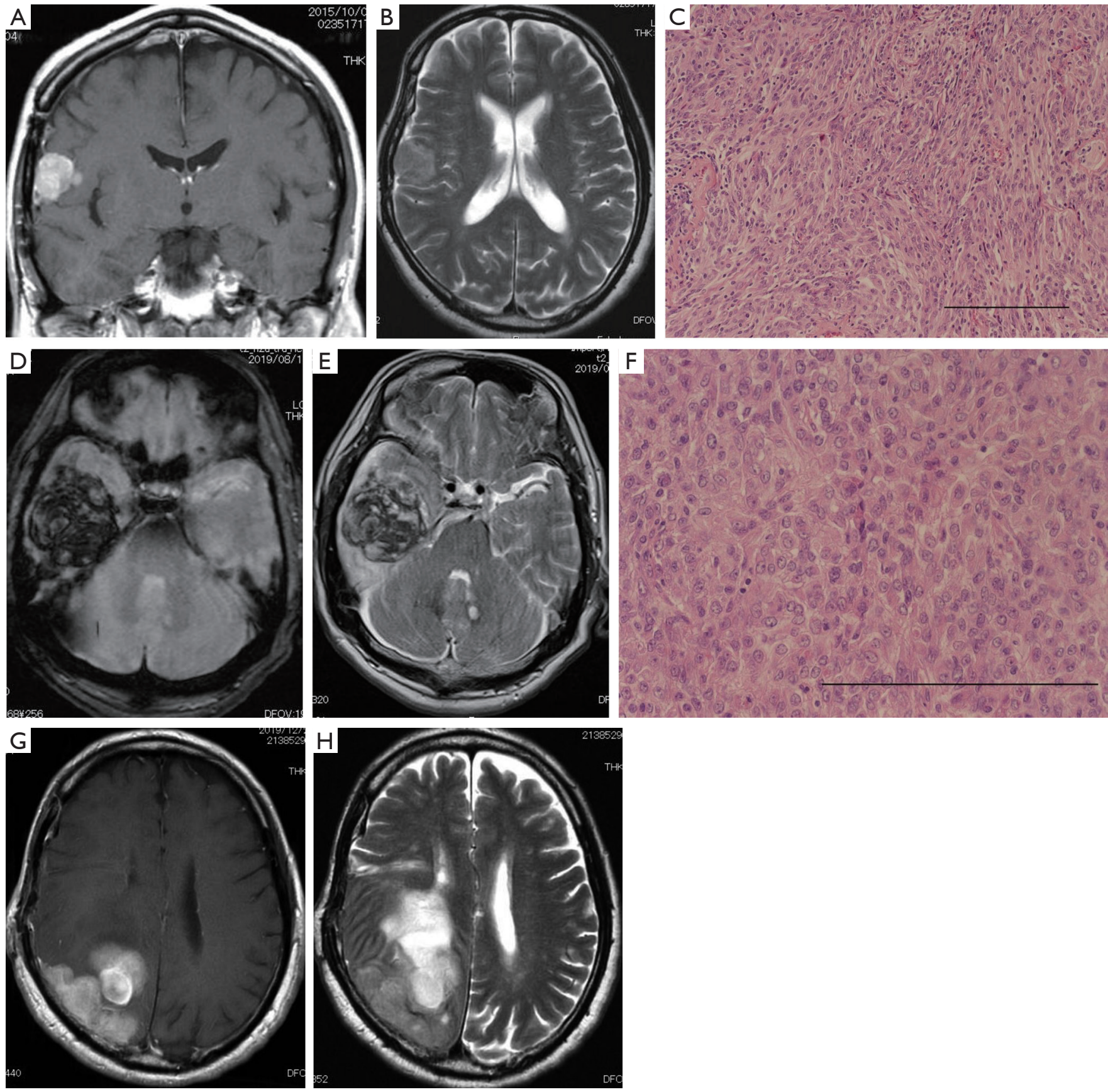

Figure 4 Preoperative magnetic resonance imaging (MRI) and histological findings of KS03 (case 3). Preoperative gadolinium-enhanced MRI (A) and T2-weighted MRI (B) at the second recurrence (KS03B) showed a homogenous enhanced tumor in the right frontal lobe. Histological findings of the initial tumor (KS03A) (C). Tumor cells were arranged in a sheet with an onion-like structure and diagnosed as meningothelial meningioma (WHO grade I) (hematoxylin \& eosin staining: 200×, scale bar =200 $\mu$ m). Preoperative diffusion weighted (D) and T2-weighted MRI (E) at the third recurrence (KS03C) showed the low-intensity mass in the right temporal lobe. Histological findings. (F) Histological imaging at the fourth recurrence (KS03D) showing mitotic cells with plump eosinophic cytoplasm diagnosed as rhabdoid meningioma; WHO grade III (hematoxylin \& eosin staining: 400×, scale bar =200 $\mu \mathrm{m}$ ). Preoperative gadolinium-enhanced MRI (G) and T2-weighted MRI $(\mathrm{H})$ at the fourth recurrence (KS03D) showed a homogenous enhanced tumor with expanding perifocal edema in the right parietal lobe. 
malignant progression were analyzed in the present study. As shown in Table 1, all the cases in the initial tumors were diagnosed as meningothelial meningioma, grade I. And the long-term latency, the tumor was recurred with malignant transformation. To investigate alterations in malignant progression of low-grade meningiomas, the difference of macrophage polarization and transcriptional factor expression in initial and recurrent tumors were compared by immunohistochemistry and real-time RT-PCR.

CD163-positive cells were increased in during malignant progression compared with initial grade I meninigiomas (Figure 5A). To confirm involvement of M1-M2 macrophage polarization during malignant progression of benign meningioma, the number of CD68- and CD163-positive cells was counted and CD163/CD68 ratios were calculated in initial and recurrent tumors (Figure $5 B$ ). In case 1 (KS01), the CD163/CD68 ratio increased after recurrence of high-grade meningioma. In case 2 (KS02), the CD163/ CD68 ratio in grade I meningoma was as high as the tumor after malignant progression, and decreased after radiation therapy for recurrence of the malignant meningioma. In case 3 (KS03), the CD163/CD68 ratio increased in grade III tumor (Figure 5B).

\section{Immunobistochemical and mRNA analysis of FOXM1}

Immunohistochemistry and mRNA expression of FOXM1 were obviously increased during malignant progression in meningiomas compared with initial benign tumor, but decreased after recurrence of high-grade tumors (Figure $6 A, B$ ). Interestingly, FOXM1 was induced in recurrent grade I meningioma (KS01B, KS03B), although initial grade I meningioma (KS03A) had undetectable FOXM1 mRNA expression. FOXM1 mRNA levels were increased by 9,4 , and 7 times in tumor samples obtained at recurrence compared with initial tumor samples from KS01, KS02, and KS03, respectively, but clearly decreased following radiation therapy (Figure 6B).

\section{Discussion}

Meningiomas are benign tumors generally treated with surgical excision. However, treatment for residual and recurrent meningiomas remains controversial, particularly concerning repeated surgical resection and additional radiotherapy. Recent clinical studies have shown a significant relationship between extent of tumor removal and recurrence-free survival and overall survival in all pathological grades of meningiomas $(24,25)$. For long-term follow-up of more than 10 years, extent of resection should be emphasized when predicting prognosis and postoperative treatment and frequency of radiologic long-term follow-up after surgery should be carefully considered (26). Even with aggressive surgical treatment of radical resection, malignant progression is inevitable.

Anaplastic meningioma constitutes 1-3\% of all meningiomas. The clinical prognosis is poor despite treatment with multiple surgical resections and adjuvant radiotherapy, with a median time to recurrence ranging between 9.6 and 42.1 months $(27,28)$. Anaplastic meningioma has histological features of malignancy with a high mitotic index, and some anaplastic meningiomas are difficult to identify as meningothelial neoplasm because they share similar features to sarcoma. In the present series, case 2 (KS02) exhibited bone metaplasia and infiltration of GFAP-positive cells (data not shown). Grade II/III meningiomas occasionally arise from malignant progression of grade I tumors, even if the initial tumor including the adjacent dura was completely removed.

As a non-surgical option, it is controversial whether radiotherapy can extend the recurrence-free survival time in patients with grade I meningiomas $(29,30)$, as well as high grade meningiomas $(31,32)$. However, few reports have suggested that overall survival in all meningioma subtypes following radiotherapy may be reduced because of radiation-induced malignant progression and patient factors, such as NF2 mutation $(33,34)$. Prediction of malignant progression of meningioma and its precise mechanism and genetic alterations remains unclear. Loss of NF2 and TERT mutation are associated with recurrence and unfavorable prognosis of meningioma. Comparison of different prognoses between de novo and secondary grade II/III meningiomas indicated that TERT promoter mutation in anaplastic meningiomas is associated with a shorter recurrence-free survival in secondary anaplastic meningiomas (35-37).

Few studies compared grade I meningiomas that did and did not progress. Comparative analyses of genetic mutations in paired samples of primary and recurrent meningiomas were described previously $(38,39)$. These groups found no enrichment for any driver mutation, and primary and recurrent tumors exhibited similar driver mutations.

To our knowledge, only one report compared grade I meningioma with and without progression by transcriptome analysis (40). The presence of NF2 fusions was not linked to prior radiation therapy as NF2 fusions 
A

Initial surgery
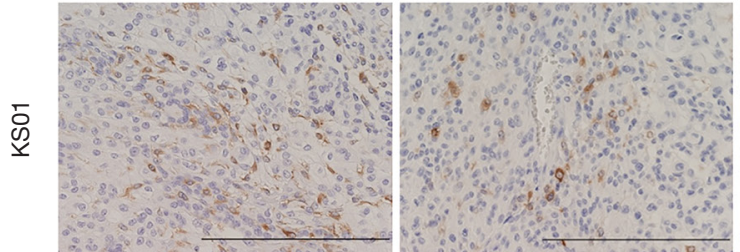

ชิ
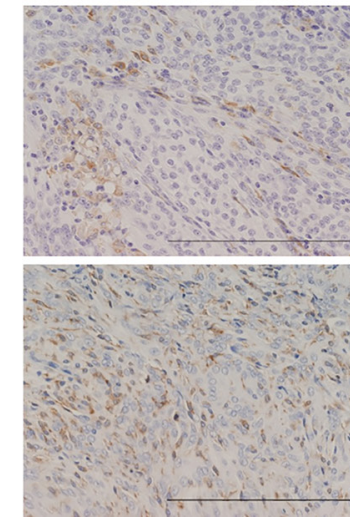

Second

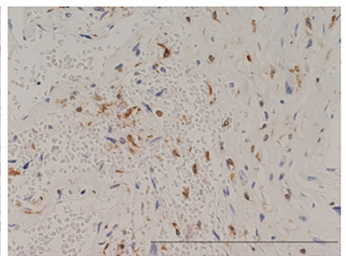

Third
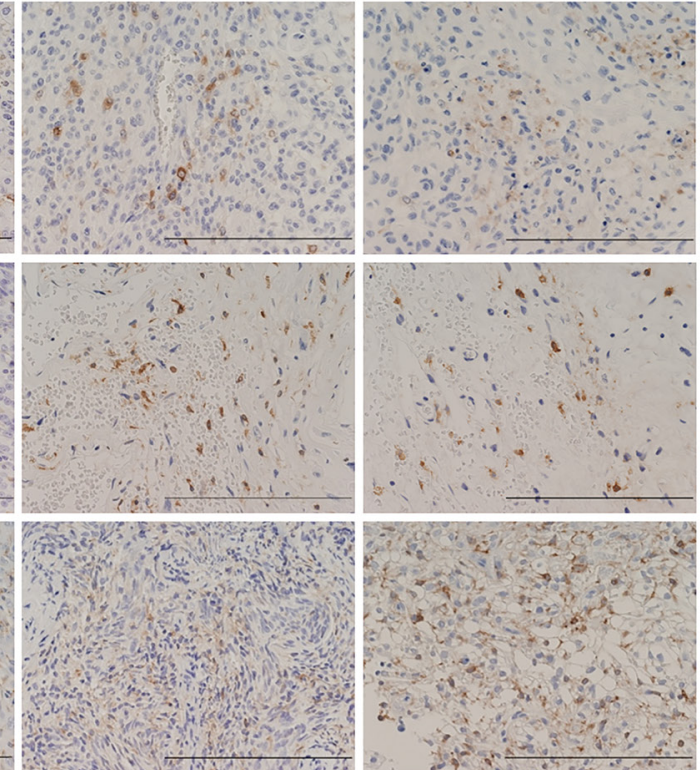

Fourth
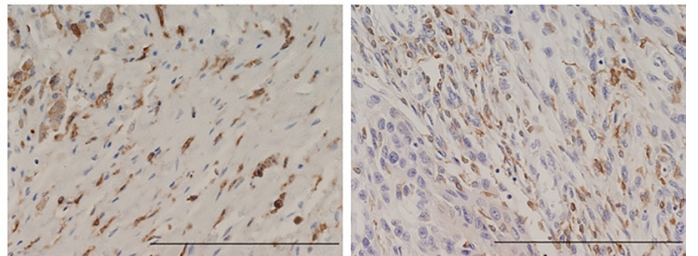

B

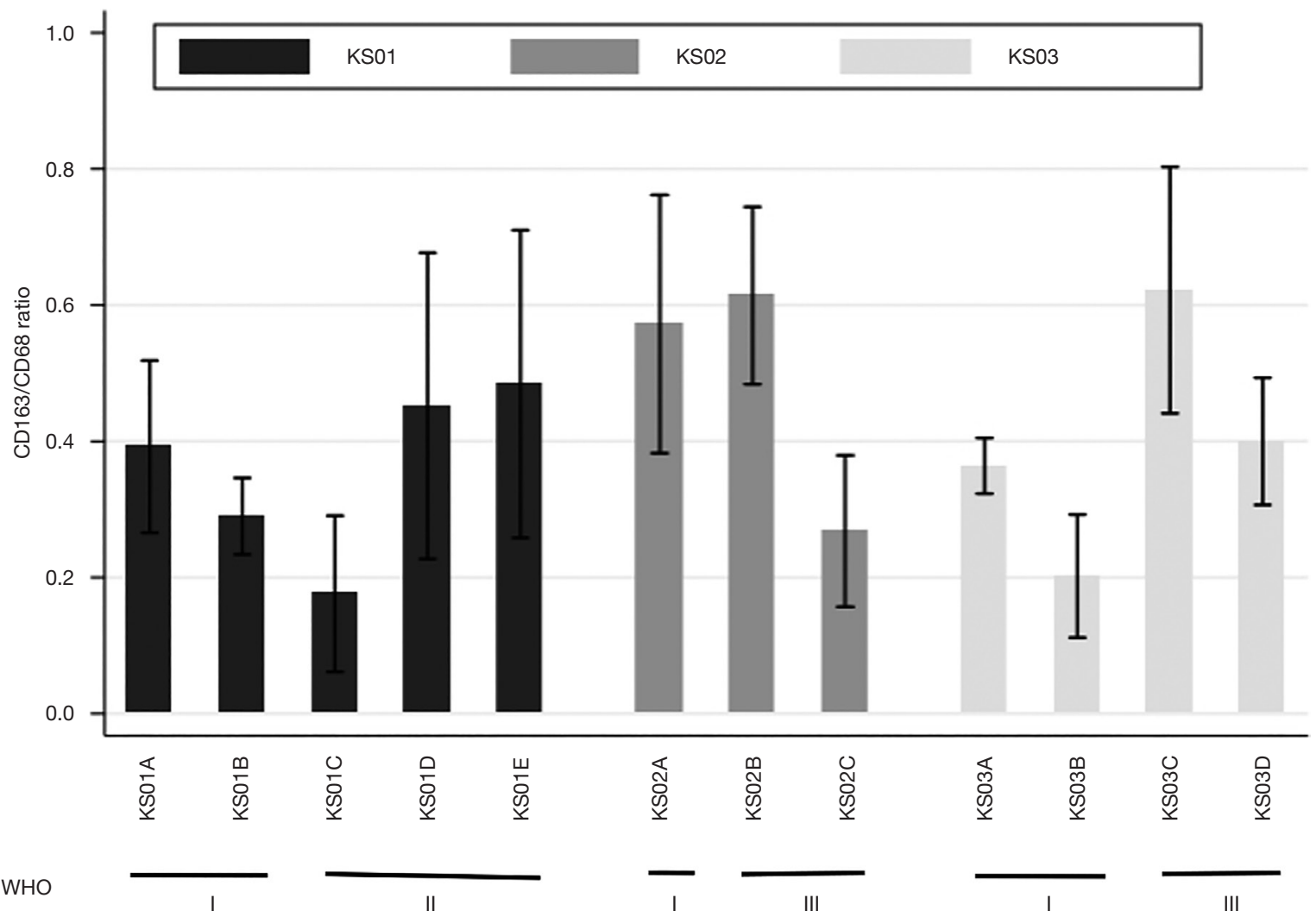

Figure 5 (A) Immunohistochemical staining of CD163 in series of tumors during multiple craniotomies from case 1 (KS01A) (upper panels), case 2 (KS02A) (middle panels), and case 3 (KS03A) (lower panels) (200×, scale bar =200 $\mu \mathrm{m})$. CD163 immunoreactivities were increased during malignant progression. (B) The ratios of CD163/CD68 expression in both grade I (KS01A-B, KS02A, KS03A-B) and recurrent grade II/III meningiomas (KS01C-E, KS02B-C, KS03C-D) were compared. 
A

A Initial surgery

Second

Third

Fourth

Fifth
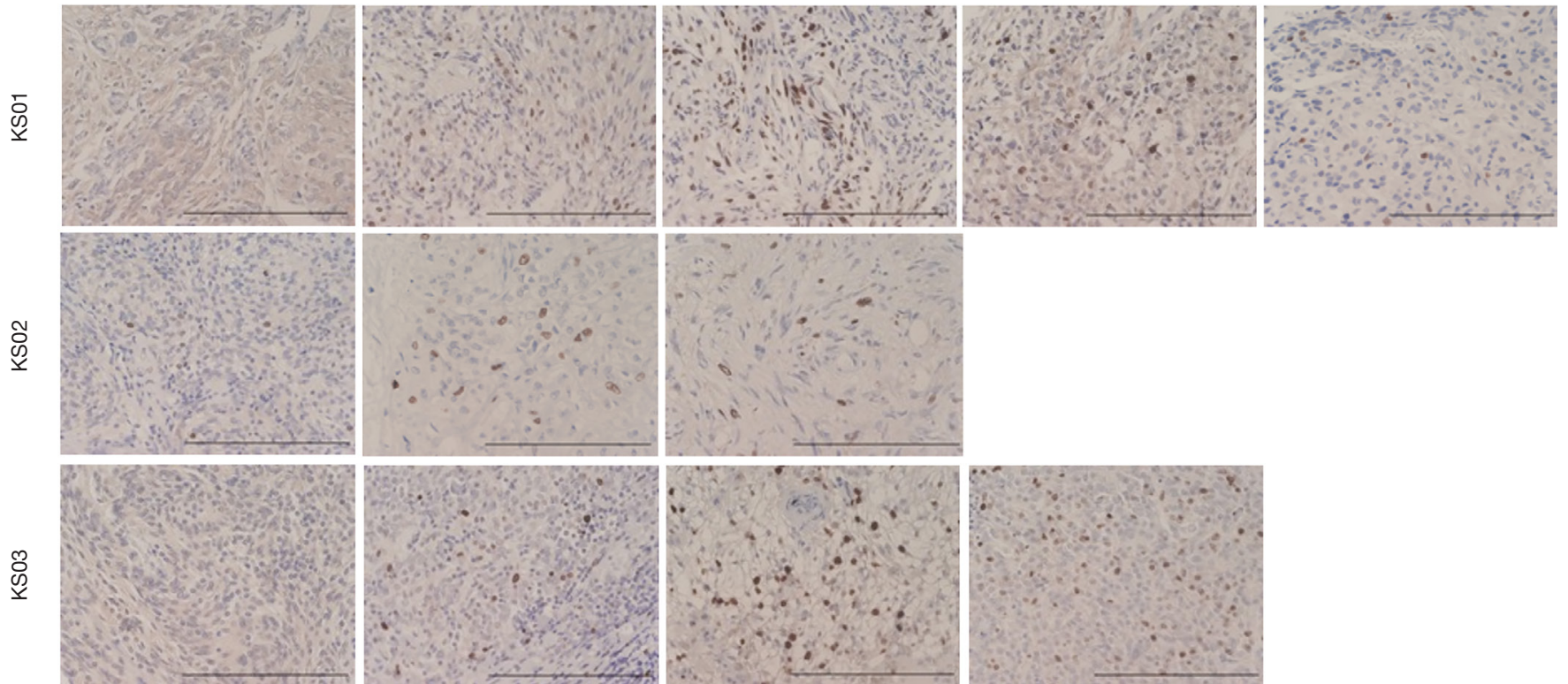

B
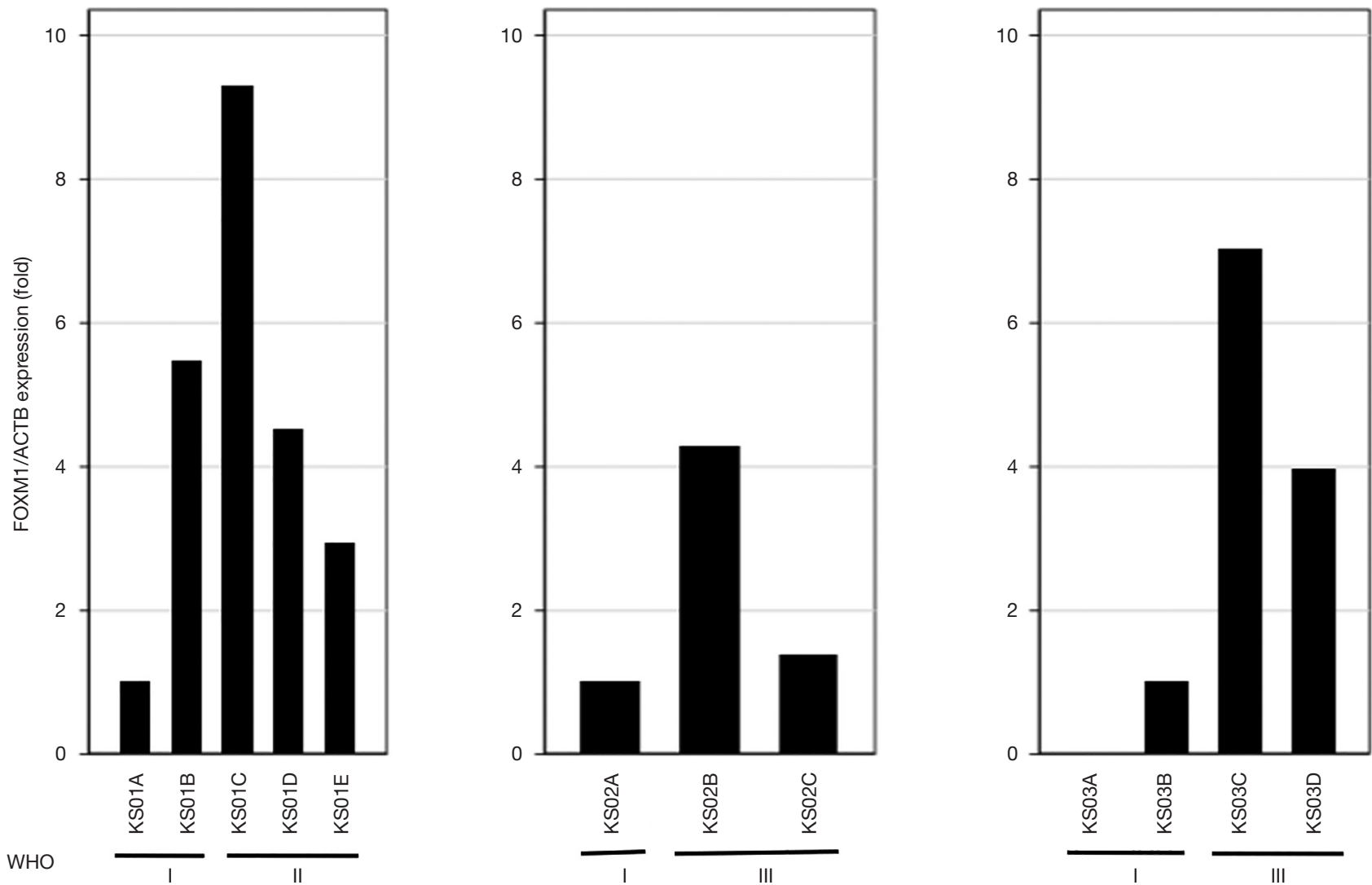

Figure 6 (A) Immunohistochemical staining of FOXM1 in series of tumors during multiple craniotomies from case 1 (KS01A) (upper panels), case 2 (KS02A) (middle panels), and case 3 (KS03A) (lower panels) (400×, scale bar $=200 \mu \mathrm{m})$. FOXM1 immunoreactivities were increased during malignant progression. (B) FOXM1 mRNA expressions in both grade I (KS01A-B, KS02A, KS03A-B) and recurrent grade II/III meningiomas (KS01C-E, KS02B-C, KS03C-D) were compared. 
were observed in patients that were naïve to radiation. Conversely, GREMLIN 2, SNORA46, and SNORA48 were highly expressed in grade I tumors compared with grade II/III tumors. The authors concluded that decreased GREMLIN 2 expression might lead to malignant behavior in meningioma.

Recently, integrated gene expression profiling studies using clinical samples from patients who had aggressive meningiomas defined by RNA sequencing, immunohistochemistry, whole-exome sequencing, DNA methylation arrays, and targeted gene expression profiling suggested that FOXM1 is a key transcription factor associated with meningioma progression and a marker for poor clinical outcomes $(11,12,38)$. The FOXM1/Wnt signaling axis in meningioma is associated with mitotic gene expression and clinical aggressiveness. In addition, FOXM1 might be a promising and an alternative therapeutic target for recurrent and refractory meningioma $(38,41)$.

In all cases in the present series, grade I meningioma, which exhibited malignant progression, revealed strong FOXM1 expression observed in recurrent grade I meningioma similar to grade II and grade III meningiomas, suggesting that FOXM1 might be a marker to predict malignant progression during grade I tumor.

In addition to genetic alteration in tumor cells with a potential role in meningioma progression, the difference in the tumor immunological microenvironment might correlate with histological grade. Thus, we evaluated M1-M2 macrophage polarization among grades I-III meningiomas. CD163 is a monocyte/macrophage lineage marker, which is associated with poor prognosis, and crosstalk between tumor cells and inflammatory cells including macrophages might be involved in meningioma progression. Macrophage polarization from M1 to M2 is influenced by the tumor microenvironment (15). In all cases in the present series, grade I meningioma that turned into malignant progression exhibited a high CD163/CD68 ratio as well as malignant progression to grade II and grade III meningiomas, suggesting that M1-M2 macrophage polarization as well as activation of FOXM1 might be a marker for predicting malignant progression. Additionally, these molecules might be potential candidates for targeted therapies for refractory meningiomas.

There are some limitations associated with the present study including the small number of paired tissues from the same patients and comparison of grade I meningioma without recurrence for more than 10 years. Prognosis of grade I meningioma is usually favorable, and disease control can be well maintained for 10 years postsurgery. However, once the tumor recurs, then tumor growth can become aggressive with subpial invasion and histological findings of malignant change. Additional therapies including multiple surgeries and adjuvant radiation might be inevitable. Aggressive surgery can decrease daily activities of living, leading to lethal status, because further surgical treatment is contraindicated due to poor performance status. Therefore, extent of resection for recurrent tumors and timing of surgery in conjunction with radiation should be cautiously considered.

Careful long-term follow-up of grade I meningiomas and recurrent meningiomas and further investigation of target molecules involved in driver mutations are important to develop a novel therapy as multidisciplinary approach for aggressive and recurrent meningiomas.

\section{Acknowledgments}

All authors greatly thank Ms. Eri Honzawa at the Division of Diagnostic Pathology, Jikei University School of Medicine Kashiwa Hospital, for technical assistance with laboratory work and sample preparation.

Funding: None.

\section{Footnote}

Reporting Checklist: The authors have completed the AME Case Series reporting checklist. Available at http://dx.doi. org/10.21037/tcr-20-1896

Conflicts of Interest: All authors have completed the ICMJE uniform disclosure form (available at http://dx.doi. org/10.21037/tcr-20-1896). The authors have no conflicts of interest to declare.

Ethical Statement: The authors are accountable for all aspects of the work in ensuring that questions related to the accuracy or integrity of any part of the work are appropriately investigated and resolved. The present study was approved by the institutional review board of our institute (permission number; 15-105 and 30-137(9158)). All procedures performed in studies involving human participants were in accordance with the ethical standards of the institutional and/or national research committee(s) and with the Helsinki Declaration (as revised in 2013). Written informed consent was obtained from the all patients. 
Open Access Statement: This is an Open Access article distributed in accordance with the Creative Commons Attribution-NonCommercial-NoDerivs 4.0 International License (CC BY-NC-ND 4.0), which permits the noncommercial replication and distribution of the article with the strict proviso that no changes or edits are made and the original work is properly cited (including links to both the formal publication through the relevant DOI and the license). See: https://creativecommons.org/licenses/by-nc-nd/4.0/.

\section{References}

1. Hortobágyi T, Bencze J, Varkoly G, et al. Meningioma recurrence. Open Med (Wars) 2016;11:168-73.

2. Louis DN, Perry A, Reifenberger G, et al. The 2016 World Health Organization Classification of Tumors of the Central Nervous System: a summary. Acta Neuropathol 2016;131:803-20.

3. Norden AD, Raizer JJ, Abrey LE, et al. Phase II trials of erlotinib o gefatnib in patients with recurrent meningioma. J Neurooncol 2010;96:211-7.

4. Nayak L, Iwamoto FM, Rudnick JD, et al. Atypical and anaplastic meningiomas treated with bevacizumab. J Neurooncol 2012;109:187-93.

5. Bi WL, Abedalthagafi M, Horowitz P, et al. Genomic landscape of intracranial meningiomas. J Neurosurg 2016;125:525-35.

6. Brastianos PK, Horowitz PM, Santagata S, et al. Genomic sequencing of meningiomas identifies oncogeneic $\mathrm{SMO}$ and AKT1 mutations. Nat Genet 2013;45:285-9.

7. Clark VE, Erson-Omay EZ, Serin A, et al. Genomic analysis of non-NF2 meningiomas reveals mutations in TRAF7, KLF4, AKT1, and SMO. Science 2013;339:1077-80.

8. Reuss DE, Piro RM, Jones DT, et al. Secretory meningiomas are defined by combined KLF4K409Q and TRAF7 mutations. Acta Neuropathol 2013;125:351-8.

9. Goutagny S, Nault JC, Mallet M, et al. High incidece of activating TERT promoter mutations in meningiomas undergoing malignant progression. Brain Pathol 2014;24:184-9.

10. Peyre M, Gauchotte G, Giry M, et al. De novo and secondary anaplastic meningioas: a study of clinical and histomolecular prognostic factors. Neuro-Oncol 2018;20:1113-21.

11. Vasudevan HN, Braunstein SE, Phillips JJ, et al. Comprehensive molecular profiling identifies FOXM1 as a key transcription factor of meningioma proliferation. Cell
Rep 2018;22:3672-83.

12. Laurendeau I, Ferrer M, Garrido D, et al. Gene expression profiling of the Hedgehog signaling pathway in human meningiomas. Mol Med 2010;16:262-70.

13. Yu G, Zhou A, Xue J, el al. FOXM1 promotes breast tumorigenesis by activating PDGF-A and forming a positive feedback loop with the PDGF/AKT signaling pathway. Oncotarget 2015;6:11281-94.

14. Quillien V, Carpentier AF, Gey A, et al. Absolute numbers of regulatory $\mathrm{T}$ cells and neutrophils in corticosteroidfree patients are predictive for response to bevacizumab in recurrent glioblastoma patients. Cancer Immunol Immunother 2019;68:871-82.

15. Tamura R, Tanaka T, Yamamoto Y, et al. Dual role of macrophage in tumor immunity. Immunotherapy 2018;10:899-909.

16. Yang C, Wei C, Wang S, et al. Elevated CD163+/CD68+ ratio at tumor invasive front is closely associated with aggressive phenotype and poor prognosis in colorectal cancer. Int J Biol Sci 2019;15:984-98.

17. Yuan X, Zhang J, Li D, et al. Prognostic significance of tumor-associated macrophages in ovarian cancer: A metaanalysis. Gynecol Oncol 2017;147:181-7.

18. Tang $X$. Tumor-associated macrophages as potential diagnostic and prognostic biomarkers in breast cancer. Cancer Lett 2013;332:3-10.

19. Komohara Y, Niino D, Saito Y, et al. Clinical significance of CD163+ tumor-associated macrophages in patients with adult T-cell leukemia/lymphoma. Cancer Sci 2013;104:945-51.

20. Salmi S, Siiskonen H, Sironen R, et al. The number and localization of CD68+ and CD163+ macrophages in different stages of cutaneous melanoma. Melanoma Res 2019;29:237-47.

21. Kong LQ, Zhu XD, Xu HX, et al. The clinical significance of the CD163+ and CD68+ macrophages in patients with hepatocellular carcinoma. PLoS One 2013;8:e59771.

22. Tamura R, Tanaka T, Ohara K, et al. Persistent restoration to the immunosupportive tumor microenvironment in glioblastoma by bevacizumab. Cancer Sci 2019;110:499-508.

23. Schindelin J, Arganda-Carreras I, Frise E, et al. Fiji: an open-source platform for biological-image analysis. Nat Methods 2012;9:676-82.

24. Nanda A, Bir SC, Maiti TK, ey al. Relevance of Simpson grading system and recurrence-free survival after surgery for World Health Organization Grade I meningioma. J Neurosurg 2017;126:201-11. 
25. Oya S, Kawai K, Nakatomi H, Saito N. Significance of Simpson grading system in modern meningioma surgery: integration of the grade with MIB-1 labeling index as a key to predict the recurrence of WHO Grade I meningiomas. J Neurosurg 2012;117:121-8.

26. Winther TL, Torp SH. Significance of the extent of resection in modern neurosurgical practice of World Health Organization grade I meningiomas. World Neurosurg 2017;99:104-10.

27. Bruna J, Brell M, Ferrer I, et al. Ki-67 proliferative index predicts clinical outcome in patients with atypical or anaplastic meningioma. Neuropathology 2007;27:114-20.

28. Pasquier D, Bijmolt S, Veninga T, et al. Atypical and malignant meningioma: outcome and prognostic factors in 119 irradiated patients. A multicenter, retrospective study of the Rare Cancer Network. Int J Radiat Oncol Biol Phys 2008;71:1388-93.

29. Wegner RE, Hasan S, Abel S, et al. Linear acceleratorbased stereotactic radiotherapy for low grade meningiomas: Improved local control with hyperfractionation. J Cent Nerv Syst Dis 2019;11:1179573519843880.

30. Gomes Dos Santos A, Solla DJF, Moscardi R, et al. Adjuvant radiotherapy did not reduced recurrence of World Health Organization grade I meningiomas with venous sinus involvement: A propensity score adjusted analysis and literature review. World Neurosurg 2019;130:e1015-e1019.

31. Sun SQ, Hawasil AH, Huang J, et al. An evidence-based treatment algorithm for the management of grade II and III meningiomas. Neurosurg Focus 2015;38:E3.

32. Zaher A, Matter MA, Zayed DH, et al. Atypical meningioma: A study of prognostic factors. World Neurosurg 2013;80:549-53.

Cite this article as: Takei J, Tanaka T, Teshigawara A, Tochigi S, Hasegawa Y, Murayama Y. Alteration of FOXM1 expression and macrophage polarization in refractory meningiomas during long-term follow-up. Transl Cancer Res 2021;10(1):553-566. doi: $10.21037 /$ tcr-20-1896
33. Agnihotri S, Suppiah S, Tonge PD, et al. Therapeutic radiation for childhood cancer drives structural aberrations of NF2 in meningiomas. Nat Commun 2017;8:186.

34. Sahm F, Schrimpf D, Stichel D, et al. DNA methylationbased classification and grading system for meningioma: a multicentre, retrospective analysis. Lancet Oncol 2017;18:682-94.

35. Goutagny S, Nault JC, Mallet M, et al. High incidence of activating TERT promoter mutations in meningiomas undergoing malignant progression. Brain Pathol 2014;24:184-9.

36. Peyre M, Gauchotte G, Giry M, et al. De novo and secondary anaplastic meningiomas: a study of clinical and histomolecular prognostic factors. Neuro-Oncol 2018;20:1113-21.

37. Sahm F, Schrimpf D, Olar A, et al. TERT promoter mutations and risk of recurrence in meningioma. J Natl Cancer Inst 2015;108:djv377.

38. Kim H, Park KJ, Ryu BK, et al. Forkhead box M1 (FOXM1) transcription factor is a key oncogenic driver of aggressive human meningioma progression. Neuropathol Appl Neurobiol 2020;46:125-41.

39. Lowenstern J, Rutland J, Gill C, et al. Comparative genomic analysis of driver mutations in matched primary and recurrent meningiomas. Oncotarget 2019;10:3506-17.

40. Viaene AN, Zhang B, Martinez-Lage M, et al. Transcriptome signatures associated with meningioma progression. Acta Neuropathol Commun 2019;7:67.

41. Radhakrishnan SK, Bhat UG, Hughes DE, et al. Identification of a chemical inhibitor of the oncogenic transcription factor forkhead box M1. Cancer Res 2006;66:9731-5. 